\title{
Neutrino Scattering Physics and Experiments at Super Beams and Neutrino Factories
}

\author{
Jorge G. Morfín ${ }^{\mathrm{a}}$

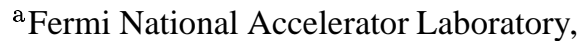 \\ P.O. Box 500, Batavia, IL, \\ 60510
}

A careful study of neutrino scattering physics is an essential part of the program to answer many open questions being addressed by several different physics communities. A deeper understanding of nuclear effects induced by neutrinos and considerably more accurate measurements of neutrino exclusive cross sections is crucial for minimizing systematics of neutrino oscillation experiments. In addition, the knowledge gained by neutrino scattering experiments is important to the Nuclear Physics and Astro-Particle physics communities as well. A review of where we expect to be in the study of neutrino scattering physics at the start of a Super Neutrino Beam Facility as well as the types of beams and detectors needed at such a facility are discussed.

\section{Introduction}

What are the open questions in neutrino physics? According to the multi-divisional study on the physics of neutrinos they are the following:

- What are the masses of the neutrinos?

- What is the pattern of mixing among the different types of neutrinos?

- Are neutrinos their own antiparticles?

- Do neutrinos violate the symmetry CP?

- Are there "sterile" neutrinos?

- Do neutrinos have unexpected or exotic properties?

- What can neutrinos tell us about the models of new physics beyond the Standard Model?

The answer to almost every one of these questions involves understanding how neutrinos interact with matter! This point was emphasized by the APS study in the summary of their study when they indicated "determination of the neutrino reaction and production cross sections required for a precise understanding of neutrino-oscillation physics and the neutrino astronomy of astrophysical and cosmological sources.and cosmological sources. Our broad and exacting program of neutrino physics is built upon precise knowledge of how neutrinos interact with matter."

A Super Neutrino Beam Facility (SNBF), with high-intensity neutrino and antineutrino beams, will offer a unique opportunity to explore neutrino scattering processes with unprecedented precision and contribute greatly to answering the above questions. While there will have been significant progress made in this area with currently running and near-future experiments, existing beams will lack the intensity needed to make the precision measurements required for complete understanding of the physics.

Neutrino scattering at a SNBF will also bring several physics communities together. The Particle physics community will be motivated by increased understanding of physics relevant to neutrino oscillation experiments The Nuclear physics community will be motivated by understanding of physics complementary to the Jlab program such as form factors, quark-hadron duality, nuclear effects and structure of nucleon. Finally the Astro-particle physics community wants to understand the role of neutrinos in stellar evolution and cosmology.

In particular, the requirements on the neutrino scattering program from the particle physics community are quite stringent. For high-statistics $\nu_{\mu}$ neutrino os- 
cillation disappearance studies, such as the MINOS experiment currently running at Fermilab, we need accurate measurements of nuclear effects with neutrinos for a neutrino energy calibration.

For upcoming $\nu_{e}$ appearance experiments a careful measurement of pion production cross sections, both coherent and resonant, are essential. Measurement of high-y cross sections are also important. The most challenging requirement of these appearance experiments in determining $\mathrm{CP}$ violation and mass hierarchy is a careful control of $\nu$ to $\bar{\nu}$ systematics at the 1 $\%$ level or better.

The pre-SNBF experiments will provide measurements of neutrino charged-current (CC) and neutralcurrent (NC) (quasi)elastic scattering and $\mathrm{CC}$ and $\mathrm{NC}$ production of pions and strange particles on nuclear targets. It will remain for the SNBF to make highprecision measurements of these processes with antineutrino beams and with nucleon targets.

A short review of what we expect to be the state of knowledge of neutrino scattering physics and what will still be awaiting experimentation at SNBF is presented below.

\subsection{Low-energy Neutrino Cross-sections: Quasi- elastic Scattering}

MINER $\nu \mathrm{A}$ will have measured [2] the crosssection up to $\mathrm{E}_{\nu}=20 \mathrm{GeV}$ with statistical errors ranging from $\leq 1 \%$ at low $\mathrm{E}_{\nu}$ up to $4 \%$ at $\mathrm{E}_{\nu}=$ $20 \mathrm{GeV}$. The expected beam systematic error is 4 $6 \%$ thanks to precision measurements of hadron production (the largest uncertainty in predicting neutrino flux) by the current MIPP experiment [1]. For the axial-vector form-factor, measurement of neutrino quasi-elastic scattering is the most direct way to improve our knowledge. MINER $\nu$ A's ability to measure $d \sigma / d Q^{2}$ to high $Q^{2}$ will have allowed investigation of the non-dipole component of the axial-vector form factor to an unprecedented accuracy. At the lower energy range of the cross section, both the K2K [3] and MiniBooNe [4] experiments will have published their measured cross sections of quasi elastic scattering also on carbon. Similar accuracy for $\bar{\nu}$ and measurements of the quasi elastic cross section off of nucleons can only be achieved with SNBF.

\subsection{Low-energy Neutrino Cross-sections: Reso- nance Production \\ To simulate resonance-mediated reactions, Monte-} Carlo programs still use early theoretical predictions by Rein \& Sehgal [8] or results from electroproduction experiments, since existing data on neutrino-induced resonance production is inadequate. Only recently have new models for resonance production by neutrinos been released by Sato \& Lee [9] and Paschos \& Lalakulich [10]. The experimental picture of the resonance and transition regions is far more obscure than the quasi-elastic and DIS regions which border it. Analysis of resonance production in MINER $\nu \mathrm{A}$ [11] will have focused on several experimental channels, including inclusive scattering in the resonance region $(W<2 \mathrm{GeV})$ and exclusive charged and neutral pion production. This channel may need additional investigation with an even-more fine-grained detector such as a LAr TPC. The investigation of this channel for $\nu$ is not limited by beam intensity but rather by detector techniques. Similar accuracy for $\bar{\nu}$ can only be achieved with SNBF.

\subsection{Low-energy Neutrino Cross-sections: Coher- ent Pion Production}

Recently the $\mathrm{K} 2 \mathrm{~K}$ experiment released a very surprising finding in that they could detect no evidence for charged-current coherent pion production at an average energy of $1.3 \mathrm{GeV}$ [12]. This is quite unexpected since the Rein-Sehgal model and earlier measurements of neutral current pion production are consistent with a much higher charged current coherent pion production cross section. MiniBooNe is currently studying this process at similar (slightly lower) energies and will release their findings soon. MINER $\nu \mathrm{A}$, with its high statistics and variety of nuclear targets, will check the $\mathrm{K} 2 \mathrm{~K}$ result and will have greatly improved our experimental understanding of coherent processes by the time of a SNBF. Figure 1 shows the estimated statistical precision of MINER $\nu$ A's CC coherent scattering measurement, as a function of neutrino energy, after background subtraction. The model of Rein \& Seghal [13] has been assumed. Also plotted are the only currently available measurements in this kinematic region showing their total errors.

MINER $\nu$ A's CC coherent event sample will also 


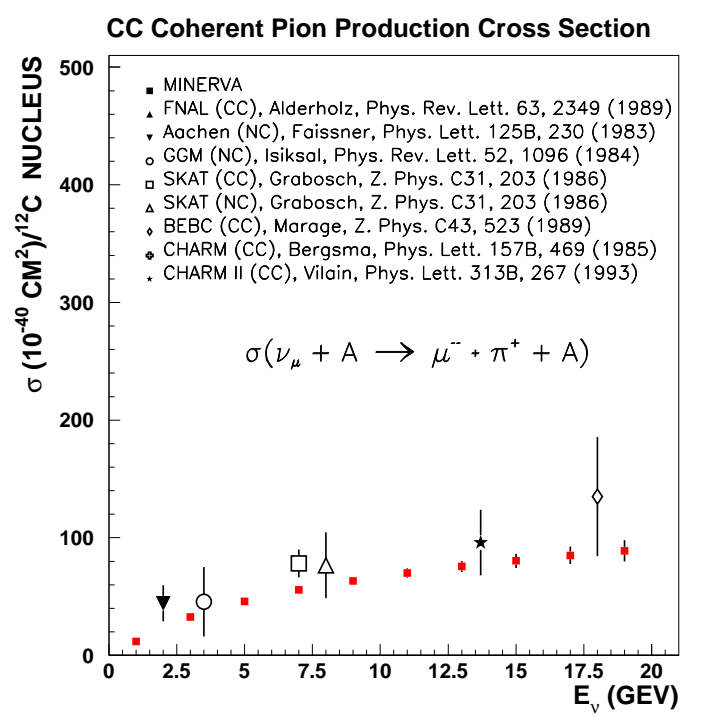

Figure 1. MINER $\nu$ A's simulated CC coherent crosssection measurement, assuming a 4-year run, statistical errors only, compared with published data.

have been used to study the differential crosssections. Comparison of the overall rates of $\mathrm{NC}$ and $\mathrm{CC}$ production, as well as the pion energy and angular distributions will allow valuable tests of the various models. For several recent models, the predicted $\mathrm{NC} / \mathrm{CC}$ ratios in coherent scattering differ by around $20 \%[13,14]$.

MINER $\nu \mathrm{A}$ will also have compared the reaction rates for lead, iron and carbon. The A dependence of the cross-section depends mainly on the assumed model of the hadron-nucleus interaction and serves as a crucial test for that component of the predictions [16].

The MINER $\nu \mathrm{A}$ results [15] will have eliminated several models for coherent production by the time a SNBF comes on-line. Similar accuracy for $\bar{\nu}$ can only be achieved with SNBF.

\subsection{Nuclear Effects in Neutrino Scattering}

Analysis of neutrino reactions with nuclear media requires understanding the nuclear environment's effect on the process [18]. There are two general cate- gories of such nuclear effects:

- The neutrino interaction probability on nuclei is modified relative to free nucleons. Nuclear effects of this type have been extensively studied in DIS structure function measurements using muon and electron beams, but have not been explored with neutrinos. Depending on the kinematic region, these nuclear effects can be quite different for neutrinos, particularly the shadowing phenomenon [17].

- Hadrons produced in a nuclear target may undergo final-state interactions (FSI), including re-scattering and absorption. These effects may significantly alter the observed final-state configuration and measured energy [22,23], and are sizable at neutrino energies typical of current and planned oscillation experiments [19].

The hadron shower observed in neutrino experiments is actually the convolution of these two effects. FSI effects are dependent on the specific final states that, even for free protons, differ for neutrino and charged-lepton reactions. The suppression or enhancement of particular final states by nuclear effects also differ for neutrino and charged lepton reactions. For these reasons, measurements of nuclear effects with charged leptons cannot be simply applied to neutrino-nucleus interactions.

It has recently been suggested that, for a given $\mathrm{Q}^{2}$, shadowing can occur at much lower energy transfer $(\nu)$ for neutrinos than for charged leptons. This effect is unaccounted for in neutrino event generators. As explained in [20], for a given $Q^{2}$ the cross-section suppression due to shadowing occurs for much lower energy transfer $(\nu)$ in neutrino interactions than for charged leptons. More recently, Kulagin \& Petti [19] have constructed a model of nuclear effects in neutrino interactions that takes account of all phenomena and produces the predicted ratio of structure functions measured off a heavy nucleus compared to an average nucleon. Figure 2 shows the predicted difference between neutrino and charged lepton shadowing as a function of the energy transfer $(\nu)$ for the ratio of iron to deuterium. Clearly this is an important effect, and without MINER $\nu$ A, there are no data available to measure it.

MINER $\nu \mathrm{A}$ will have carefully studied these effects with targets of carbon, iron and lead [20]. 


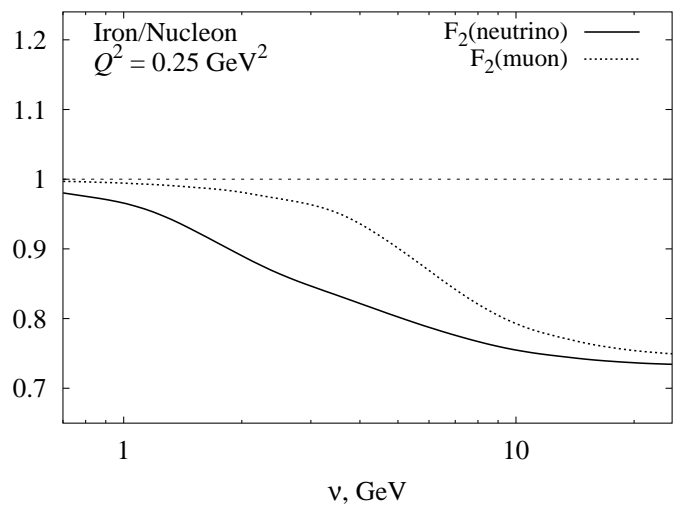

Figure 2. Predicted shadowing effects at $Q^{2}=0.25$ $\mathrm{GeV}^{2}$ as a function of energy transfer $(\nu)$, for neutrinos (solid line) and muons (dotted line) for iron compared to deuterium.

What will be missing is a comparison with deuterium which is essential for maximal understanding of these effects. Similar accuracy for $\bar{\nu}$ can only be achieved with SNBF.

\subsection{The Perturbative - Non-Perturbative Inter- face and Deep-Inelastic Scattering}

Despite the apparent dichotomy between the partonic and hadronic regimes, in nature there exist instances where the low-energy behavior of crosssections (averaged over appropriate energy intervals) closely resembles that at asymptotically high energies, calculated in terms of quark-gluon degrees of freedom. This phenomenon is referred to as quarkhadron duality and is the focus of substantial recent interest in probing the structure of the nucleon [2529]. For example, there are over 10 related experiments at JLab.

Understanding this transition requires reliable data in three kinematic regimes: in the scaling domain of high $Q^{2}$ DIS scattering; in the hadronic region of resonances and quasi-elastic scattering; and, perhaps most importantly, in the moderate $Q^{2}$ region between the two, where the transition is most dramatically manifest. MINER $\nu \mathrm{A}$ will have addressed this compelling topic for the first time with neutrinos with measurements spanning all three regimes, providing reliable data in the crucial transition region [30].

\section{Goals of a Neutrino Scattering Physics Pro- gram in the SNBF Era}

As shown, low-to-medium energy $\nu$ - nucleus scattering will be quite well covered. However $\bar{\nu}-$ nucleus and $\nu / \bar{\nu}$ - nucleon, in this same important energy range, will still not have been covered as needed. The simple reason why these topics will not have been covered is the meager event rate associated with them. The $\bar{\nu}$ event rate is down a factor of (3 - 5), depending on energy range, compared to a $\nu$ exposure. This comes from a combination of the cross-section ratio and the production rate ratio of $\pi^{+}$to $\pi^{-}$. Combining this factor with the low absolute cross-section associated with low-energy neutrinos and what would take a 3 year run to accumulate with $\nu$ would take $\mathbf{9}$ - 15 years with $\bar{\nu}$. Similarly a neutrino scattering physics program on the nucleon (Liquid $\mathrm{H}_{2}$ and $\mathrm{D}_{2}$ targets) has an event rate an order-of-magnitude lower than with a carbon target. To completely understand $\nu / \bar{\nu}$ nucleon as well as $\bar{\nu}$ - nucleus scattering physics, the higher statistics available with a SNBF is essential.

\subsection{Neutrino Beam Requirements}

The standard 2-horn beam provides a very pure $\nu$ beam with only a small admixture of $\bar{\nu}$ background. Unfortunately, the converse is not true. As an example, the NuMI 2-horn $\bar{\nu}$ le-beam actually yields more $\nu$ events than $\bar{\nu}$ events. This is due to the forward going higher energy positive pions that go right through the neck of the horns and, thus experience no deflecting magnetic field. For a high-precision $\bar{\nu}$ beam in the SNBF era the logical choice of beam would be a signselected beam such as was used by Fermilab experiment E-815 (NuTeV) [31]. With this sign-selected beam, the $\nu$ contamination of the $\bar{\nu}$ beam is reduced to $4 \times 10^{-3}$, a dramatic improvement compared to the 2-horn beam.

\subsection{Detector Requirements}

An important goal of an SNBF neutrino scattering program will be a careful study of $\nu / \bar{\nu}$ - nucleon scattering. This will require a large liquid hydrogen/deuterium target. The challenge will be to know what is happening to the events produced within the hydrogen/deuterium target before they leave the tar- 
get and enter the tracking detectors surrounding the target. A way to record the tracks within the cryogenic liquid target itself is make the target active as in a Bubble Chamber. Contemporary large bubble chambers are being developed for WIMP searches by a University of Chicago/Fermilab collaboration and for Bubble Chamber spectroscopy by Los Alamos lab. These new chambers use CCD coupled readout to directly transfer the image to disk. Patern recognition and tracking software developed for emulsion experiments can then be directly employed to reproduce the three-dimensional images.

\section{REFERENCES}

1. Y. Fisyak et al. [The MIPP Collaboration], "P907: Proposal to Measure Particle Production in the Meson Area Using Main Injector Primary and Secondary Beams", proposal to the FNAL PAC, May 2000.

2. A. Bodek, H. Budd, "Quasi-Elastic Scattering”, MINER $\nu$ A Note 100, September, 2004. http: //minerva.fnal.gov

3. R. Gran [K2K Collaboration], Nucl. Phys. Proc. Suppl. 139, 54 (2005).

4. J. Monroe [MiniBooNE Collaboration], Nucl. Phys. Proc. Suppl. 139, 59 (2005) [arXiv:hepex/0408019].

5. N. J. Baker et al., Phys. Rev. D23, 2499 (1981).

6. T. Kitagaki et al., Phys. Rev. D26, 436 (1983).

7. K.L. Miller et al., Phys. Rev. D26 (1982) 537.

8. D. Rein and L. M. Sehgal, Annals Phys. 133, 79 (1981).

9. T. Sato and T. S. Lee, Int. J. Mod. Phys. A 20, 1668 (2005)

10. O. Lalakulich and E. A. Paschos, Phys. Rev. D 71, 074003 (2005)

11. O. Lalakulich, E. Paschos, S. Wood. "The Study of Resonance Production in the MINER $\nu \mathrm{A}$ Experiment", MINER $\nu$ A Note 200, September, 2004. http://www.pas.rochester.edu/minerva/

12. M. Hasegawa et al. [K2K Collaboration], arXiv:hep-ex/0506008.

13. D. Rein and L. M. Sehgal, Nucl. Phys. B223, 29 (1983).

14. E. A. Paschos and A. V. Kartavtsev, (2003), hep$\mathrm{ph} / 0309148$.
15. H. Gallagher, D. Harris, A. Kartavtsev, and E. Paschos, "Neutral and Charged Current Neutrino-Nucleus Coherent Measurements", MINER $\nu$ A Note 300, October, 2004. http://www.pas.rochester.edu/minerva/

16. E. Paschos and A. Kartavtsev (private communication).

17. B.Z. Kopeliovich, hep-ph/0409079.

18. MINER $\nu$ A Collaboration, op. cit., pgs. 99 - 108, $192-200$.

19. E. A. Paschos, M. Sakuda, I. Schienbein and J. Y. Yu, arXiv:hep-ph/0408185, (2004).

20. S. Boyd, S. Kulagin, J. G. Morfin and R. Ransome, "Studying Neutrino-induced $\mathrm{Nu}$ clear Effects with the MINER $\nu \mathrm{A}$ Detector". MINER $\nu$ A Note 700, September, 2004. http://www.pas.rochester.edu/minerva/

21. S. A. Kulagin and R. Petti, "Global study of nuclear structure functions," arXiv:hep$\mathrm{ph} / 0412425$.

22. M.K. Jones et al., Phys. Rev. C48, 2800 (1993); R.D. Ransome et al., Phys. Rev. C46, 273 (1992); R.D. Ransome et al., Phys. Rev. C45, R509 (1992).

23. D. Rowntree et al., Phys. Rev. C60, 054610 (1999); B. Kotlinksi et al., Eur. Phys. J. A9, 537 (2000).

24. MINER $\nu$ A Collaboration, op. cit., pgs. 83 - 94.

25. Sabine Jeschonnek and J.W. Van Orden, Phys. Rev. D69:540,2004.

26. F. E. Close and Nathan Isgur, Phys. Lett. B509:81-86, 2001.

27. I. Niculescu et al, Phys. Rev. Lett. 85:11821185,2000 .

28. I. Niculescu et al., Phys. Rev. Lett. 85 (2000) 1186.

29. R. Ent, W Melnitchouk, and C .E. Keppel, "Quark-Hadron Duality in Electron Scattering", submitted to Physics Reports.

30. C. E. Keppel and I. Niculescu. "Studying the Perturbative / Non-Perturbative QCD Interface with the MINER $\nu \mathrm{A}$ Detector". MINER $\nu \mathrm{A}$ Note 500, September, 2004. verb+http://www.pas.rochester.edu/minerva/+

31. M. H. Shaevitz, S. R. Mishra, F. Sciulli, R. H. Bernste in, F. Borcherding, M. Lamm and F. Taylor, FERMILAB-PROPOSAL-0815, FNAL-TM 1884 